PREPARED FOR THE U.S. DEPARTMENT OF ENERGY, UNDER CONTRACT DE-AC02-76CH03073

PPPL-3960

PPPL-3960

UC-70

\author{
Analytic, High-beta Solutions \\ of the Helical Grad-Shafranov Equation
}

by

D.R. Smith and A.H. Reiman

May 2004

$\left.\stackrel{M}{M}\right|_{\substack{\text { PRInCETON PLASIMA } \\ \text { PHYSICS LABORATORY }}} ^{D}$

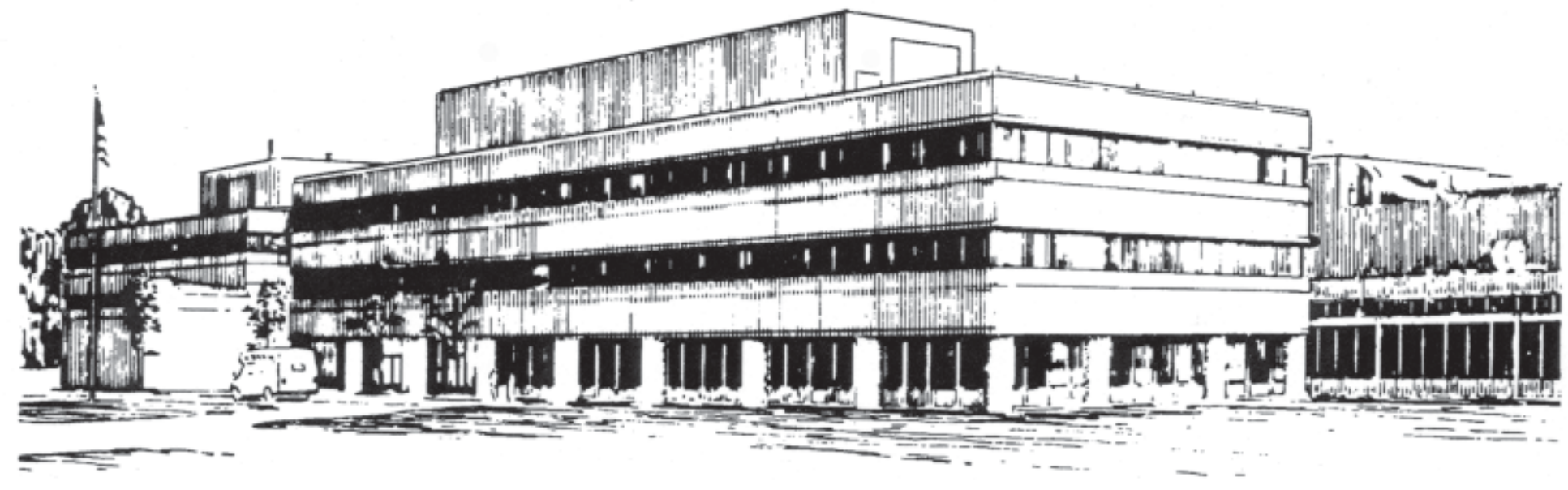

PRINCETON PLASMA PHYSICS LABORATORY PRINCETON UNIVERSITY, PRINCETON, NEW JERSEY 


\section{PPPL Reports Disclaimer}

This report was prepared as an account of work sponsored by an agency of the United States Government. Neither the United States Government nor any agency thereof, nor any of their employees, makes any warranty, express or implied, or assumes any legal liability or responsibility for the accuracy, completeness, or usefulness of any information, apparatus, product, or process disclosed, or represents that its use would not infringe privately owned rights. Reference herein to any specific commercial product, process, or service by trade name, trademark, manufacturer, or otherwise, does not necessarily constitute or imply its endorsement, recommendation, or favoring by the United States Government or any agency thereof. The views and opinions of authors expressed herein do not necessarily state or reflect those of the United States Government or any agency thereof.

\section{Availability}

This report is posted on the U.S. Department of Energy's Princeton Plasma Physics Laboratory Publications and Reports web site in Fiscal Year 2004. The home page for PPPL Reports and Publications is: http://www.pppl.gov/pub_report/

DOE and DOE Contractors can obtain copies of this report from:

U.S. Department of Energy

Office of Scientific and Technical Information

DOE Technical Information Services (DTIS)

P.O. Box 62

Oak Ridge, TN 37831

Telephone: (865) 576-8401

Fax: (865) 576-5728

Email: reports@adonis.osti.gov

This report is available to the general public from:

National Technical Information Service

U.S. Department of Commerce

5285 Port Royal Road

Springfield, VA 22161

Telephone: $1-800-553-6847$ or

(703) $605-6000$

Fax: (703) 321-8547

Internet: http://www.ntis.gov/ordering.htm 


\title{
Analytic, high-beta solutions of the helical Grad-Shafranov equation
}

\author{
D. R. Smith and A. H. Reiman \\ Princeton Plasma Physics Laboratory, Princeton, New Jersey 08543
}

(Dated: April 26, 2004)

\begin{abstract}
We present analytic, high-beta $(\beta \sim O(1))$, helical equilibrium solutions for a class of helical axis configurations having large helical aspect ratio, with the helix assumed to be tightly wound. The solutions develop a narrow boundary layer of strongly compressed flux, similar to that previously found in high beta tokamak equilibrium solutions. The boundary layer is associated with a strong localized current which prevents the equilibrium from having zero net current.
\end{abstract}




\section{INTRODUCTION}

Helically symmetric MHD equilibria represent the large aspect ratio limit of stellarator equilibria, and they have been extensively used to study the physics of large aspect ratio stellarator configurations. (See e.g. Refs. 1-7 and references cited therein.) Recent analytical studies of the helical equilibrium equations in the context of astrophysical problems have focused on finding exact solutions for special pressure and current profiles that linearize the equilibrium equation. ${ }^{89}$ In this paper we use an asymptotic matching technique to obtain analytic solutions for a class of high $\beta$ helical equilibria, valid for a broad class of pressure and current profiles. Of particular interest is the value of the net current in the equilibrium solutions.

The asymptotic matching technique applied here is an extension of that developed by Cowley et al for high $\beta$, large aspect ratio, axisymmetric equilibria. ${ }^{10} \mathrm{~A}$ new piece of physics that appears in non-axisymmetric equilibria is the generation of rotational transform (finite $\iota=1 / q)$ by the non-axisymmetric flux surface geometry even in the absence of a current. This allows conventional stellarator equilibria to have zero net current in each flux surface (the steady state solution in the absence of a bootstrap current and externally driven current), while axisymmetric equilibria cannot have zero net current. As we will discuss below, for the class of equilibria we have studied, the high beta equilibrium solutions develop a narrow boundary layer of strongly compressed flux, similar to that previously found in high $\beta$ tokamak equilibrium solutions. The boundary layer is associated with a strong localized current which prevents the equilibrium from having zero net current.

In this paper we specialize to helical axis configurations having a large helical excursion. To further simplify the calculation, the helix is assumed to be tightly wound. (In the limit that the winding becomes infinitely tight, we recover the solution to the axisymmetric GradShafranov equation.) These assumptions will be stated more precisely in the next section. We study fixed boundary equilibria, with the boundary shape assumed to be circular. The generalization to a non-circular boundary is straightforward using the method of Ref. 10 . It should be noted that the free-boundary solution can be expected to introduce additional constraints on realizable equilibria. ${ }^{11}$

Section II develops the helical Grad-Shafranov equation in dimensionless form and introduces our ordering. Section III presents an analytic solution of the helical Grad-Shafranov 
equation for $\beta=O(1)$ and $q_{h}=O(1)$, where $q_{h}$ is defined in Section II. The net current is shown to be non-vanishing, with the current localized primarily in the boundary layer. Section IV considers other possible orderings, further investigating the conditions under which a boundary layer forms and the implications for the existence of equilibrium solutions with zero net current.

\section{HELICAL GRAD-SHAFRANOV EQUATION AND ORDERING}

Consider a helical plasma with helical excursion $r_{0}$ and minor radius $a$ in a cylindrical coordinate system $r, \theta, z$ as shown in Figure 1. The plasma is helically symmetric if physical quantities are functions only of $\mathrm{r}$ and $u \equiv m \theta-k z$ for some fixed $m$ and $k . m=0$ corresponds to the special case of axisymmetry. We will focus primarily on the $m=1$ helical system in this paper, but will retain $m$ throughout the analysis to facilitate comparison with the axisymmetric case. The helical plasma is periodic in the $z$ direction with wavelength $2 \pi / k$ and consists of $N$ periods. The vectors $\mathbf{u}$ and $\mathbf{h}$ are defined as ${ }^{1,2}$

$$
\begin{array}{r}
\mathbf{u} \equiv \frac{m \hat{\theta}-k r \hat{\mathbf{z}}}{m^{2}+k^{2} r^{2}} \\
\mathbf{h} \equiv \hat{\mathbf{r}} \times \mathbf{u}=\frac{m \hat{\mathbf{z}}+k r \hat{\theta}}{m^{2}+k^{2} r^{2}} .
\end{array}
$$

The divergence-free magnetic field can be written

$$
\mathbf{B}=\nabla \psi \times \mathbf{h}+B_{h} \mathbf{h}
$$

where $\psi=\psi(r, u)$ is the helical stream function and $B_{h} \equiv m B_{z}+k r B_{\theta}$ is the helical magnetic field. Ampere's law $\mu_{0} \mathbf{J}=\nabla \times \mathbf{B}$ gives

$$
\mu_{0} \mathbf{J}=\nabla B_{h} \times \mathbf{h}-\left(\left(m^{2}+k^{2} r^{2}\right) \mathcal{L}(\psi)-\frac{2 m k B_{h}}{m^{2}+k^{2} r^{2}}\right) \mathbf{h}
$$

where

$$
\mathcal{L}(\psi) \equiv \frac{1}{r} \frac{\partial}{\partial r}\left(\frac{r}{m^{2}+k^{2} r^{2}} \frac{\partial \psi}{\partial r}\right)+\frac{1}{r^{2}} \frac{\partial^{2} \psi}{\partial u^{2}}
$$

The force balance equation is $\nabla p=\mathbf{J} \times \mathbf{B}$. From $\mathbf{B} \cdot \nabla p=0$ we find $p=p(\psi)$. From $\mathbf{J} \cdot \nabla p=0$ we find $B_{h}=B_{h}(\psi)$. Substituting $\mathbf{B}$ and $\mathbf{J}$ into the force balance equation gives the helical Grad-Shafranov equation ${ }^{1,2}$

$$
\frac{1}{r} \frac{\partial}{\partial r}\left(\frac{r}{m^{2}+k^{2} r^{2}} \frac{\partial \psi}{\partial r}\right)+\frac{1}{r^{2}} \frac{\partial^{2} \psi}{\partial u^{2}}=-\mu_{0} \frac{d p(\psi)}{d \psi}-\frac{B_{h}(\psi) \frac{d B_{h}(\psi)}{d \psi}}{m^{2}+k^{2} r^{2}}+\frac{2 m k B_{h}(\psi)}{\left(m^{2}+k^{2} r^{2}\right)^{2}} .
$$


The standard derivation of the axisymmetric Grad-Shafranov equation can be recovered by setting $m=0$ in Eqs. 1-6, with $\mathbf{h} \rightarrow \hat{\theta} / k r$ and $B_{h} \rightarrow k r B_{\theta}$. It follows from Eq. 4 that in axisymmetry the net current is determined entirely by $\mathcal{L}(\psi)$. The net current for a nontrivial axisymmetric equilibrium cannot be set to zero. For non-axisymmetric equilibria the net current is also influenced by the value of $B_{h}(\psi)$, allowing the net current to be set to zero on each flux surface in conventional stellarator equilibria. A key issue investigated in this paper is the impact of the $B_{h}$ component of the current at high $\beta$, focusing particularly on the conditions under which it is possible to obtain zero net current at high $\beta$.

The helical Grad-Shafranov equation requires that the pressure profile $p(\psi)$ and the helical field profile $B_{h}(\psi)$ be specified. Rather than specify $B_{h}(\psi)$ directly, we will specify the helical safety factor profile $q_{h}(\psi)$ and relate $q_{h}(\psi)$ to $B_{h}(\psi)$. The toroidal flux is defined $\mathrm{as}^{3}$

$$
\begin{aligned}
\Phi_{T}(\psi) & \equiv \frac{k}{2 \pi} \int_{V} \mathbf{B} \cdot \hat{\mathbf{z}} d^{3} x \\
& =\frac{k}{2 \pi} \int_{V} \nabla \psi \cdot(\mathbf{h} \times \hat{\mathbf{z}}) d^{3} x+\frac{k}{2 \pi} \int_{V} \frac{m B_{h}}{m^{2}+k^{2} r^{2}} d^{3} x
\end{aligned}
$$

where the integral is taken over the volume of one period. The helical flux is defined $\mathrm{as}^{3}$

$$
\begin{aligned}
\Phi_{H}(\psi) & \equiv \int_{0}^{2 \pi} \int_{r\left(\psi_{\max }\right)}^{r(\psi)} \frac{m^{2}+k^{2} r^{2}}{k} \mathbf{B} \cdot \mathbf{u} d r d \theta \\
& =-\frac{2 \pi}{k}\left(\psi-\psi_{\max }\right)
\end{aligned}
$$

where the integral is taken over a ribbon of constant $u$ that extends from the magnetic axis $r\left(\psi_{\max }\right)$ to the flux surface $r(\psi)$ and twists with the magnetic axis. The helical safety factor is defined $\mathrm{as}^{3}$

$$
q_{h}(\psi) \equiv \frac{d \Phi_{T}}{d \Phi_{H}}=-\frac{k}{2 \pi} \Phi_{T}^{\prime}(\psi)
$$

The toroidal flux derivative $\Phi_{T}^{\prime}(\psi)$ can be expressed as ${ }^{3,4}$

$$
\Phi_{T}^{\prime}(\psi)=\frac{k}{2 \pi} \int_{V} \frac{2 k m^{2}}{\left(m^{2}+k^{2} r^{2}\right)^{2}} d^{3} x+\frac{k}{2 \pi} \frac{d}{d \psi} \int_{V} \frac{m B_{h}}{m^{2}+k^{2} r^{2}} d^{3} x
$$

The differential volume element is

$$
d^{3} x=\frac{|d S| d \psi}{|\nabla \psi|}=\sqrt{\left(\left.\frac{\partial r}{\partial u}\right|_{\psi}\right)^{2}\left(1+\frac{k^{2} r^{2}}{m^{2}}\right)+\frac{r^{2}}{m^{2}}} \frac{d u d z d \psi}{|\nabla \psi|} .
$$


In the axisymmetric limit, the helical flux reduces to the poloidal flux and the helical safety factor reduces to the conventional safety factor. The corresponding large aspect ratio stellarator has a rotational transform

$$
\frac{\iota}{2 \pi}=\frac{N\left(1+q_{h}\right)}{m q_{h}} .
$$

It is convenient to work with dimensionless variables. The dimensionless radial variable $x$ is defined such that

$$
r \equiv r_{0}+a x=r_{0}(1+\epsilon x)
$$

where $\epsilon \equiv a / r_{0}$ is the inverse helical aspect ratio and $-1 \leq x \leq 1$. (Note that $\epsilon$ is not the inverse aspect ratio of the corresponding large aspect ratio stellarator.) We consider configurations for which $\epsilon \ll 1$, using $\epsilon$ as an expansion parameter in subsequent analysis. The winding parameter is $\kappa \equiv k a$. The plasma column cannot overlap itself and this imposes the geometric constraint $\kappa \leq \pi$ for non-axisymmetric equilibria. We specialize further to configurations for which the helix is tightly wound, $\kappa \gg \epsilon$, such that $\kappa=O(1)$. $\psi$ is normalized as

$$
\psi=\psi_{\max } \Psi
$$

such that $0 \leq \Psi \leq 1$. The boundary conditions are $\Psi=1$ at the magnetic axis and $\Psi=0$ at the circular wall. Similarly, $p(\psi)$ and $B_{h}(\psi)$ are normalized as

$$
\begin{aligned}
p & =p_{\max } P \\
B_{h} & =\frac{\kappa}{\epsilon} B_{\max } B
\end{aligned}
$$

such that $0 \leq P, B \leq O(1)$. With these dimensionless variables, the helical Grad-Shafranov equation is

$$
\begin{array}{r}
\epsilon^{2}\left[\frac{1}{1+\epsilon x} \frac{\partial}{\partial x}\left(\frac{1+\epsilon x}{\epsilon^{2} m^{2}+\kappa^{2}(1+\epsilon x)^{2}} \frac{\partial \Psi}{\partial x}\right)+\frac{1}{(1+\epsilon x)^{2}} \frac{\partial^{2} \Psi}{\partial u^{2}}\right]= \\
-\frac{\mu_{0} a^{2} p_{\max }}{\psi_{\max }^{2}} \frac{d P}{d \Psi}-\frac{a^{2} B_{\max }^{2}}{\psi_{\max }^{2}} \frac{\kappa^{2} B \frac{d B}{d \Psi}}{\epsilon^{2} m^{2}+\kappa^{2}(1+\epsilon x)^{2}}+\frac{a B_{\max }}{\psi_{\max }} \frac{2 m \kappa^{2} \epsilon^{3} B}{\left(\epsilon^{2} m^{2}+\kappa^{2}(1+\epsilon x)^{2}\right)^{2}} .
\end{array}
$$

Scalings relating $\psi_{\max }, p_{\max }$, and $B_{\max }$ are now needed.

Combining Eqs. 11-13 and utilizing dimensionless variables gives

$$
\begin{aligned}
q_{h}(\Psi)=- & \frac{1}{2 \pi} \int_{1}^{\Psi} \int_{u_{\min }(\varphi)}^{u_{\max }(\varphi)} \frac{2 m \kappa^{2} \epsilon^{3}(1+\epsilon x)}{\left(\epsilon^{2} m^{2}+\kappa^{2}(1+\epsilon x)^{2}\right)^{2}} \sqrt{1+\left(\kappa^{2}+\frac{\epsilon^{2} m^{2}}{(1+\epsilon x)^{2}}\right)\left(\left.\frac{\partial x}{\partial u}\right|_{\varphi}\right)^{2}} \frac{d u d \varphi}{|\bar{\nabla} \varphi|} \\
& -\frac{1}{2 \pi} \frac{a B_{\max }}{\psi_{\max }} \int_{u_{\min }(\Psi)}^{u_{\max }(\Psi)} \frac{\kappa^{2} B(\Psi)(1+\epsilon x)}{\epsilon^{2} m^{2}+\kappa^{2}(1+\epsilon x)^{2}} \sqrt{1+\left(\kappa^{2}+\frac{\epsilon^{2} m^{2}}{(1+\epsilon x)^{2}}\right)\left(\left.\frac{\partial x}{\partial u}\right|_{\Psi}\right)^{2}} \frac{d u}{\mid \nabla \Psi} \mid
\end{aligned}
$$


where $\bar{\nabla}=a \nabla$ and $u_{\min / \max }(\Psi)$ are the minimum and maximum values of $u$ on the flux surface $\Psi$. For the purposes of Section 3, we will take

$$
\frac{a B_{\max }}{\psi_{\max }}=1
$$

so the first term of Eq. 20 is $O\left(\epsilon^{3}\right)$ and the second term is $O(1) . q_{h}$ can now be written

$$
q_{h}(\Psi)=-\frac{\kappa^{2} B(\Psi)}{2 \pi} \int_{u_{\min }(\Psi)}^{u_{\max }(\Psi)} \frac{1+\epsilon x}{\epsilon^{2} m^{2}+\kappa^{2}(1+\epsilon x)^{2}} \sqrt{1+\left(\kappa^{2}+\epsilon^{2} m^{2}\right)\left(\left.\frac{\partial x}{\partial u}\right|_{\Psi}\right)^{2}} \frac{d u}{|\bar{\nabla} \Psi|}+O\left(\epsilon^{3}\right)
$$

such that $q_{h}=O(1)$. The ordering $\beta=O(1)$ is satisfied by simply taking $\mu_{0} p_{\max }=B_{\max }^{2}$. This $\beta$ ordering combined with Eq. 21 gives

$$
\frac{\mu_{0} a^{2} p_{\max }}{\psi_{\max }^{2}}=1
$$

With the scalings of Eqs. 21 and 23, the helical Grad-Shafranov equation is now

$$
\begin{array}{r}
\epsilon^{2}\left[\frac{1}{1+\epsilon x} \frac{\partial}{\partial x}\left(\frac{1+\epsilon x}{\epsilon^{2} m^{2}+\kappa^{2}(1+\epsilon x)^{2}} \frac{\partial \Psi}{\partial x}\right)+\frac{1}{(1+\epsilon x)^{2}} \frac{\partial^{2} \Psi}{\partial u^{2}}\right]= \\
-\frac{d P}{d \Psi}-\frac{\kappa^{2} B \frac{d B}{d \Psi}}{\epsilon^{2} m^{2}+\kappa^{2}(1+\epsilon x)^{2}}+\frac{2 m \kappa^{2} \epsilon^{3} B}{\left(\epsilon^{2} m^{2}+\kappa^{2}(1+\epsilon x)^{2}\right)^{2}} .
\end{array}
$$

\section{ANALYTIC SOLUTION OF THE HELICAL GRAD-SHAFRANOV EQUA-} TION FOR $\beta=O(1)$ AND $q_{h}=O(1)$

In this section we use an asymptotic matching technique introduced by Cowley et $\mathrm{al}^{10}$ to solve the helical Grad-Shafranov equation for $\beta=O(1)$ and $q_{h}=O(1)$. Our assumptions have been described in the previous section.

\section{A. Core Solution}

In the plasma core (outer region), derivatives of $\Psi$ are $O(1)$ and the helical GradShafranov equation can be written

$$
0=(1+2 \epsilon x) \frac{d P}{d \Psi}+G(\Psi)+O\left(\epsilon^{2}\right)
$$

where

$$
G(\Psi) \equiv B(\Psi) \frac{d B}{d \Psi}
$$


$P(\Psi)$ and $q_{h}(\Psi)$ are specified, so $\Psi, B(\Psi)$, and $G(\Psi)$ are expanded in powers of $\epsilon$ and then all functions of $\Psi$ are Taylor expanded around $\Psi_{0}$. These expansions are substituted into the helical Grad-Shafranov equation and orders of $\epsilon$ are collected. The $O(1)$ equation gives $0=P^{\prime}\left(\Psi_{0}\right)+G_{0}\left(\Psi_{0}\right)$ or

$$
P\left(\Psi_{0}\right)+\frac{1}{2} B_{0}^{2}\left(\Psi_{0}\right)=C
$$

where $C$ is a constant of integration. The $O(\epsilon)$ equation gives

$$
0=2 x P^{\prime}\left(\Psi_{0}\right)+G_{1}\left(\Psi_{0}\right)
$$

and therefore $\Psi_{0}=\Psi_{0}(x)$. In fact, all $\Psi_{n}$ are independent of $u$. This functional form can satisfy the boundary condition $\Psi=1$ on the magnetic axis, but not the boundary condition $\Psi=0$ on the circular wall. A boundary layer is needed to satisfy the wall boundary condition. The boundary layer is located at the wall, so the outer solution is physically located in the core away from the wall.

Now use Eq. 22 to find $\Psi_{0}(x)$. With derivatives of $\Psi$ large in the boundary layer and $\Psi_{0}$ independent of $u$ in the core, Eq. 22 gives

$$
\begin{aligned}
q_{h}\left(\Psi_{0}\right) & =-\frac{B\left(\Psi_{0}\right)}{2 \pi \frac{d \Psi_{0}}{d x}} \int_{-\kappa \sqrt{1-x^{2}}}^{\kappa \sqrt{1-x^{2}}} d u+O(\epsilon) \\
& =-\frac{\kappa}{\pi} \sqrt{2\left(C-P\left(\Psi_{0}\right)\right)} \frac{\sqrt{1-x^{2}}}{\frac{d \Psi_{0}}{d x}}+O(\epsilon)
\end{aligned}
$$

where we used $u_{\max / \min }= \pm \kappa \sqrt{1-x^{2}}$ and Eq. 27. See Figures 1 and 2 in Ref. 10. Integration with respect to $x$ from -1 to $x$ gives

$$
\int_{D}^{\Psi_{0}} \frac{q_{h}(\varphi) d \varphi}{\sqrt{2(C-P(\varphi))}}=-\frac{\kappa}{2 \pi}\left(\frac{\pi}{2}+x \sqrt{1-x^{2}}+\sin ^{-1} x\right) .
$$

The constants $C$ and $D$ will be determined by boundary layer properties. Given $P(\Psi)$ and $q_{h}(\Psi)$, Eq. 31 provides an implicit relation between $\Psi_{0}$ and $x$ valid only in the core region.

\section{B. Boundary Layer Solution}

In the boundary layer, derivatives of $\Psi$ are large. With this in mind, the helical GradShafranov equation can be written

$$
\epsilon^{2}\left[\frac{\partial^{2} \Psi}{\partial x^{2}}+\kappa^{2} \frac{\partial^{2} \Psi}{\partial u^{2}}\right]=-\kappa^{2}(1+2 \epsilon x) \frac{d P}{d \Psi}-\kappa^{2} G(\Psi)+O\left(\epsilon^{2}\right) .
$$


To simplify the analysis, we take the plasma boundary to be circular in planes of constant $\theta$. (The solution can be extended to boundaries of arbitrary shape using the method of Ref. 10.) The geometry suggests analysis in a polar coordinate system $\rho, \zeta$ where $x=\rho \cos \zeta$ and $u=\kappa \rho \sin \zeta$. The derivatives transform accordingly,

$$
\begin{aligned}
\frac{\partial}{\partial x} & =\cos \zeta \frac{\partial}{\partial \rho}-\frac{\sin \zeta}{\rho} \frac{\partial}{\partial \zeta} \\
\kappa \frac{\partial}{\partial u} & =\sin \zeta \frac{\partial}{\partial \rho}+\frac{\cos \zeta}{\rho} \frac{\partial}{\partial \zeta} .
\end{aligned}
$$

Transforming to polar coordinates, the helical Grad-Shafranov equation becomes

$$
\epsilon^{2}\left[\frac{\partial^{2} \Psi}{\partial \rho^{2}}+\frac{1}{\rho} \frac{\partial \Psi}{\partial \rho}+\frac{1}{\rho^{2}} \frac{\partial^{2} \Psi}{\partial \zeta^{2}}\right]=-\kappa^{2}(1+2 \epsilon \rho \cos \zeta) \frac{d P}{d \Psi}-\kappa^{2} G(\Psi)+O\left(\epsilon^{2}\right) .
$$

To match the core solution and also match the boundary condition at $\rho=1$, the left hand side must be $O(\epsilon)$. This is achieved with a boundary layer width $\delta=O\left(\epsilon^{1 / 2}\right)$. Define the boundary layer variable $t$ such that $\rho \equiv 1-\epsilon^{1 / 2} t$ to get

$$
\epsilon \frac{\partial^{2} \Psi}{\partial t^{2}}-\epsilon^{3 / 2} \frac{\partial \Psi}{\partial t}=-\kappa^{2}\left(1+2 \epsilon\left(1-\epsilon^{1 / 2} t\right) \cos \zeta\right) \frac{d P}{d \Psi}-\kappa^{2} G(\Psi)+O\left(\epsilon^{2}\right)
$$

Now expand $\Psi$ and $G$ in powers of $\epsilon^{1 / 2}$ and then Taylor expand around $\Psi_{0}$. The $O(1)$ equation reproduces Eq. 27. The $O\left(\epsilon^{1 / 2}\right)$ equation gives $G_{1 / 2}\left(\Psi_{0}\right)=0$. The $O(\epsilon)$ equation gives

$$
\frac{\partial^{2} \Psi_{0}}{\partial t^{2}}=2 \kappa^{2}\left(x\left(\Psi_{0}\right)-\cos \zeta\right) P^{\prime}\left(\Psi_{0}\right)
$$

where $x\left(\Psi_{0}\right)$ comes from Eq. 31. Multiply Eq. 37 by $\frac{\partial \Psi_{0}}{\partial t}$ and integrate to get

$$
\frac{1}{2}\left(\frac{\partial \Psi_{0}}{\partial t}\right)^{2}=2 \kappa^{2} \int_{\Psi_{c o r e}(\cos \zeta)}^{\Psi_{0}}(x(\varphi)-\cos \zeta) P^{\prime}(\varphi) d \varphi \equiv \kappa^{2} V\left(\Psi_{0}, \zeta\right)
$$

where $\Psi_{\text {core }}(\cos \zeta)$ is the core solution given by Eq. 31. Further integration of Eq. 38 gives

$$
t\left(\Psi_{0}, \zeta\right)=\int_{0}^{\Psi_{0}} \frac{d \varphi}{\sqrt{2 \kappa^{2} V(\varphi, \zeta)}} .
$$

Eq. 39 can be inverted to give the boundary layer solution, $\Psi_{0}(\rho, \zeta)$ with $\rho=1-\epsilon^{1 / 2} t$.

We can now determine the constants $C$ and $D$ in Eq. 31. The boundary layer width goes to zero at $x=-1$, so it follows that $D=0$. The boundary condition $\Psi_{0}=1$ at the magnetic axis, $x=1-O\left(\epsilon^{1 / 2}\right)$, gives

$$
\int_{0}^{1} \frac{q_{h}(\varphi) d \varphi}{\sqrt{2(C-P(\varphi))}}=-\frac{\kappa}{2}
$$

which determines $C$. 


\section{Net Current}

We evaluate the current within a flux surface using Ampere's law and a contour in the $\theta=$ const plane,

$$
\mu_{0} I=\oint \mathbf{B} \cdot \mathbf{d} \mathbf{l}=\left.\int_{0}^{2 \pi} \mathbf{B} \cdot \frac{\partial \mathbf{x}}{\partial \zeta}\right|_{\theta} d \zeta
$$

where $\mathbf{x}=r \hat{\mathbf{r}}+z \hat{\mathbf{z}}$. In the core region, the integrand can be evaluated to give

$$
\left.\mathbf{B} \cdot \frac{\partial \mathbf{x}}{\partial \zeta}\right|_{\theta}=-\frac{\epsilon \psi_{\max }}{\kappa}\left[x \frac{\partial \Psi}{\partial x}+m x B(\Psi)+O(\epsilon)\right] .
$$

For the boundary layer region, transform to the polar coordinates $\rho, \zeta$ to get

$$
\left.\mathbf{B} \cdot \frac{\partial \mathbf{x}}{\partial \zeta}\right|_{\theta}=-\frac{\epsilon \psi_{\max }}{\kappa}\left[\rho \frac{\partial \Psi}{\partial \rho}+m \rho B(\Psi) \cos \zeta+O(\epsilon)\right] .
$$

With $\rho=1-\epsilon^{1 / 2} t$

$$
\left.\mathbf{B} \cdot \frac{\partial \mathbf{x}}{\partial \zeta}\right|_{\theta}=\frac{\psi_{\max }}{\kappa}\left[\epsilon^{1 / 2} \frac{\partial \Psi}{\partial t}+O(\epsilon)\right]
$$

so the boundary layer contribution to Eq. 41 dominates the core contribution. The normalized current within a flux surface can be written using Eq. 38 to give

$$
\frac{\mu_{0} I}{\psi_{\max }}=\epsilon^{1 / 2} \int_{B L} \sqrt{2 V\left(\Psi_{0}, \zeta\right)} d \zeta+O(\epsilon)
$$

where the integral is taken over the boundary layer portion of the flux surface. The normalized net current is given by the current within the outermost flux surface,

$$
\begin{aligned}
\frac{\mu_{0} I}{\psi_{\max }} & =\epsilon^{1 / 2} \int_{0}^{2 \pi} \sqrt{2 V\left(\Psi_{0}, \zeta\right)} d \zeta+O(\epsilon) \\
& =2 \epsilon^{1 / 2} \int_{0}^{2 \pi}\left(\int_{-1}^{\cos \zeta} p\left[\psi_{\text {core }}(x)\right] d x\right)^{1 / 2} d \zeta+O(\epsilon) .
\end{aligned}
$$

The net current is finite, with an $O\left(\epsilon^{1 / 2}\right)$ normalized value.

D. Example: $P(\Psi)=P^{\prime} \Psi$ and $q_{h}(\Psi)=q_{0}$

If we assume $P(\Psi)=P^{\prime} \Psi$ and $q_{h}(\Psi)=q_{0}$, then we can explicitly evaluate Eq. 31 to recover $\Psi_{0}(x)$ in the core region. ${ }^{10}$ That is, we can solve the equation

$$
\int_{0}^{\Psi_{0}} \frac{q_{0}}{\sqrt{2\left(C-P^{\prime} \varphi\right)}} d \varphi=\frac{\kappa}{2 \pi} A(x)
$$


for $\Psi_{0}(x)$ where

$$
A(x)=\frac{\pi}{2}+x \sqrt{1-x^{2}}+\sin ^{-1} x .
$$

To determine $C$, use the boundary condition $\Psi_{0}=1$ at $x=1-O\left(\epsilon^{1 / 2}\right)$ to get

$$
C=\frac{\left(8 q_{0}^{2}+P^{\prime}\right)^{2}}{32 q_{0}^{2}} .
$$

Now Eq. 48 can be evaluated to give

$$
\Psi_{0}(x)=\frac{\kappa A(x)}{\pi}\left(1+\frac{P^{\prime}}{8 q_{0}^{2}}\right)-\frac{\kappa^{2} A(x)^{2}}{\pi^{2}} \frac{P^{\prime}}{8 q_{0}^{2}} .
$$

This is $\Psi_{0}(x)$ in the core region assuming $P(\Psi)=P^{\prime} \Psi$ and $q_{h}(\Psi)=q_{0}$. It in turn determines $\Psi_{0}(\rho, \zeta)$ in the boundary layer through Eqs. 38 and 39, given the inversion of $\Psi_{0}(x)$ to determine $x\left(\Psi_{0}\right)$.

\section{ALTERNATIVE ORDERINGS ASSOCIATED WITH A BOUNDARY LAYER}

We further investigate the conditions under which a boundary layer forms, and the implications for the existence of equilibrium solutions with zero net current. In order to obtain zero net current for a nontrivial equilibrium, the leading order contributions to the current due to $B_{h}$ and $\psi$ must cancel. We have seen that this cannot happen for $\beta=O(1)$, $q_{h}=O(1)$. In this section we consider a more general set of orderings.

With $\beta=O\left(\epsilon^{\gamma_{1}}\right)$ and $q_{h}=O\left(\epsilon^{\gamma_{2}}\right)$, the helical Grad-Shafranov equation in the core region is

$$
\epsilon^{2}\left[\frac{\partial^{2} \Psi}{\partial x^{2}}+\kappa^{2} \frac{\partial^{2} \Psi}{\partial u^{2}}\right]=-\epsilon^{2 \gamma_{2}} \kappa^{2}\left((1+2 \epsilon x) \epsilon^{\gamma_{1}} \frac{d P}{d \Psi}+G(\Psi)\right)+O\left(\epsilon^{3}\right) .
$$

If the $2 \epsilon x \frac{d P}{d \Psi}$ term of Eq. 52 is lower order than the LHS, then a boundary layer is needed to match the boundary condition $\Psi=0$ on the wall. Thus, a boundary layer is needed if

$$
2 \gamma_{2}+\gamma_{1}<1
$$

We must also have $\gamma_{1} \geq 0(\beta \leq O(1))$, or else the leading order equation gives the non-selfconsistent $d P / d \Psi=0$. The implied constraint on $\gamma_{2}$ is also sufficient for the second term of Eq. 20 to dominate the first. (We require $\gamma_{2}<3$ for this purpose.)

To interpret Eq. 53, we define an average helical beta by analogy with the poloidal beta for the axisymmetric case, $\bar{\beta}_{h} \equiv \mu_{0} p_{\max } r_{0}^{2} / \psi_{\max }^{2}$. Eq. 53 corresponds to the condition $\epsilon \overline{\beta_{h}} \gg 1$. 
Now suppose that there is a boundary layer of thickness $\delta=\epsilon^{\gamma_{3}}$. In the boundary layer, the helical Grad-Shafranov equation to lowest order in $\epsilon$ can be written

$$
\epsilon^{2\left(1-\gamma_{3}\right)} \frac{\partial^{2} \Psi}{\partial t^{2}}=-\epsilon^{2 \gamma_{2}} \kappa^{2}\left((1+2 \epsilon x) \epsilon^{\gamma_{1}} \frac{d P}{d \Psi}+G(\Psi)\right)
$$

The LHS must be of equal or lower order than the $2 \epsilon x \frac{d P}{d \Psi}$ term for the boundary layer solution to match the wall boundary condition. This requires

$$
1-2 \gamma_{3} \leq \gamma_{1}+2 \gamma_{2}
$$

Using Eq. 53, we see $\gamma_{3}>0$. The integrand of the boundary layer current (Eq. 43) to lowest order can be written

$$
\left.\mathbf{B} \cdot \frac{\partial \mathbf{x}}{\partial \zeta}\right|_{\theta}=-\frac{\epsilon \psi_{\max }}{\kappa}\left[-\epsilon^{-\gamma_{3}} \frac{\partial \Psi}{\partial t}+\epsilon^{\gamma_{2}} m B(\Psi) \cos \zeta\right]
$$

It follows from $\gamma_{3}>0$ that the boundary layer current is too large to be balanced by the current in the core. To achieve zero net current, the two terms contributing to the boundary layer current in Eq. 56 must balance, requiring $\gamma_{2}=-\gamma_{3}$. Using Eq. 55, this leads to $\gamma_{1} \geq 1$. Therefore, the net current in these types of equilibria cannot vanish for $\gamma_{1}<1$, that is, for $\beta / \epsilon \gg 1$. We conclude that a helical equilibrium of the type we are studying cannot have zero net current if $\epsilon \overline{\beta_{h}} \gg 1$ and $\beta / \epsilon \gg 1$.

The magnitude of the normalized net current in the boundary layer is determined by $\gamma_{3}$. Eqs. 53 and 55 imply that $\gamma_{3}>0 . \gamma_{3}$ can approach 0 , consistent with these constraints, if $\gamma_{1}+2 \gamma_{2}$ approaches 1 .

We note that Ref. 5 has established the existence (and MHD stability) of zero net current, loosely wound, helical axis stellarators with values of beta approaching $\mathrm{O}(1)$. (i.e. $\langle\beta\rangle$ approaching 30\%) These stellarators have helical aspect ratio of order one, and they do not have $\beta / \epsilon \gg 1$. The analysis of this paper has been done in the tightly wound limit, and does not address the existence of zero net current equilibria in loosely wound, helical axis stellarators having $\beta=O(1)$ and larger helical aspect ratio.

\section{DISCUSSION}

In this paper we have studied a class of helical axis configurations having large helical aspect ratio, with the helix assumed to be tightly wound. In Section 4 we defined an average 
helical beta by analogy with the poloidal beta for an axisymmetric equilibrium. When $\epsilon \bar{\beta}_{h} \gg 1$, the equilibrium solutions for these configurations develop a narrow boundary layer of strongly compressed flux, similar to that which develops in large aspect ratio tokamaks when $\epsilon \beta_{p} \gg 1$. The boundary layer is associated with a strong localized current.

In Section 3, we focused on the special case where $\beta=O(1)$ and $q_{h}=O(1)$, obtaining an analytic solution for the equilibrium by a boundary layer analysis. In the core of the plasma (outer region) the flux surfaces collapse to cylindrical geometry. The boundary layer allows the flux surface shape to transition to that of the plasma boundary near the edge.

For conventional stellarator equilibrium solutions, the case of zero net current is of particular interest. The current density is given by Eq. 4. It might appear that, for $m \neq 0$, we should always be able to increase the magnitude of $B_{h}$ and adjust its profile to make the net current on each flux surface vanish. However, at high $\beta$, as we increase the magnitude of $B_{h}$ relative to that of $\psi$, the boundary layer becomes increasingly compressed, causing $\mathcal{L}(\psi)$ to increase correspondingly. At these high values of $\beta$, the $\mathcal{L}(\psi)$ contribution to the current always dominates.

We find that configurations of the type studied here cannot have zero net current when $\epsilon \overline{\beta_{h}} \gg 1$ and $\beta / \epsilon \gg 1$. 


\section{Acknowledgements}

This work was supported by DOE contract DE-AC02-76CH03073. 


\section{References}

1 J. Johnson, C.R. Oberman, R.M. Kulsrud, and E.A. Frieman, Phys. Fluids 1, 281 (1958).

2 B.B. Kadomtsev, JETP 37, 962 (1960).

3 V.D. Pustovitov and V.D. Shafranov, in Reviews of Plasma Physics, edited by B.B. Kadomtsev (Consultants Bureau, New York, 1990), Vol. 15, p. 163.

4 H. Grad, P.N. Hu, and D.C. Stevens, in Proc. Nat. Acad. Sci., edited by R.L. Sinsheimer et al. (National Academy of Sciences, Washington D.C., 1975), Vol. 72, p. 3789 .

5 D.A. Monticello, R. L. Dewar, H. P. Furth, and A. H. Reiman, Phys. Fluids 27, 1248 (1984).

6 K.V. Brushlinsky, N.M. Zueva, M.S. Mikhailova, and SV Sin'ko, Plasma Physics Reports 24, 907 (1998).

7 K.V. Brushlinsky, Computer Physics Communications 126, 37 (2000).

8 L.J. Palumbo and M. Platzeck, Astrophysical Journal 416, 656 (1993).

9 O.I. Bogoyavlenskij, Letters in Mathematical Physics 51, 235 (2000).

10 S.C. Cowley, P.K. Kaw, R.S. Kelly, and R.M. Kulsrud, Phys. Fluids B 3, 2066 (1991).

11 H. Qin and A. Reiman, Phys. Plasmas 4, 762 (1997). 


\section{Figure Captions}

Figure 1: Geometry of the helical configuration with large helical aspect ratio. 
Smith, Figure 1

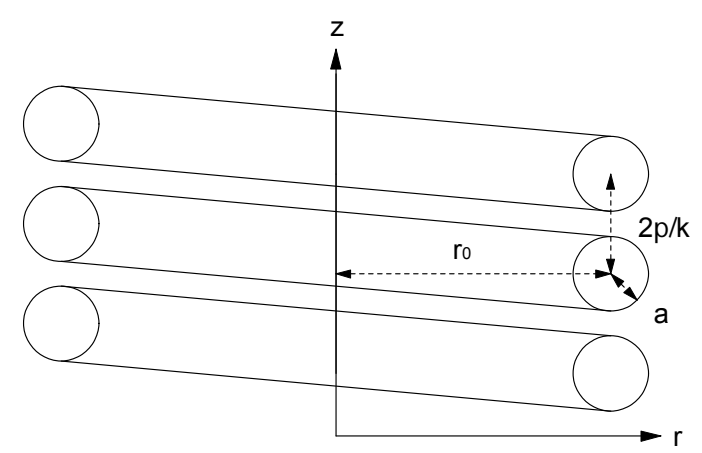




\section{External Distribution}

Plasma Research Laboratory, Australian National University, Australia

Professor I.R. Jones, Flinders University, Australia

Professor João Canalle, Instituto de Fisica DEQ/IF - UERJ, Brazil

Mr. Gerson O. Ludwig, Instituto Nacional de Pesquisas, Brazil

Dr. P.H. Sakanaka, Instituto Fisica, Brazil

The Librarian, Culham Laboratory, England

Mrs. S.A. Hutchinson, JET Library, England

Professor M.N. Bussac, Ecole Polytechnique, France

Librarian, Max-Planck-Institut für Plasmaphysik, Germany

Jolan Moldvai, Reports Library, Hungarian Academy of Sciences, Central Research Institute for Physics, Hungary

Dr. P. Kaw, Institute for Plasma Research, India

Ms. P.J. Pathak, Librarian, Institute for Plasma Research, India

Ms. Clelia De Palo, Associazione EURATOM-ENEA, Italy

Dr. G. Grosso, Instituto di Fisica del Plasma, Italy

Librarian, Naka Fusion Research Establishment, JAERI, Japan

Library, Laboratory for Complex Energy Processes, Institute for Advanced Study, Kyoto University, Japan

Research Information Center, National Institute for Fusion Science, Japan

Dr. O. Mitarai, Kyushu Tokai University, Japan

Dr. Jiangang Li, Institute of Plasma Physics, Chinese Academy of Sciences, People's Republic of China

Professor Yuping Huo, School of Physical Science and Technology, People's Republic of China

Library, Academia Sinica, Institute of Plasma Physics, People's Republic of China

Librarian, Institute of Physics, Chinese Academy of Sciences, People's Republic of China

Dr. S. Mirnov, TRINITI, Troitsk, Russian Federation, Russia

Dr. V.S. Strelkov, Kurchatov Institute, Russian Federation, Russia

Professor Peter Lukac, Katedra Fyziky Plazmy MFF UK, Mlynska dolina F-2, Komenskeho Univerzita, SK-842 15 Bratislava, Slovakia

Dr. G.S. Lee, Korea Basic Science Institute, South Korea

Institute for Plasma Research, University of Maryland, USA

Librarian, Fusion Energy Division, Oak Ridge National Laboratory, USA

Librarian, Institute of Fusion Studies, University of Texas, USA

Librarian, Magnetic Fusion Program, Lawrence Livermore National Laboratory, USA

Library, General Atomics, USA

Plasma Physics Group, Fusion Energy Research Program, University of California at San Diego, USA

Plasma Physics Library, Columbia University, USA

Alkesh Punjabi, Center for Fusion Research and Training, Hampton University, USA

Dr. W.M. Stacey, Fusion Research Center, Georgia Institute of Technology, USA

Dr. John Willis, U.S. Department of Energy, Office of Fusion Energy Sciences, USA

Mr. Paul H. Wright, Indianapolis, Indiana, USA 
The Princeton Plasma Physics Laboratory is operated by Princeton University under contract with the U.S. Department of Energy.

\author{
Information Services \\ Princeton Plasma Physics Laboratory \\ P.O. Box 451 \\ Princeton, NJ 08543
}

Phone: 609-243-2750

Fax: 609-243-2751

e-mail: pppl_info@pppl.gov

Internet Address: http://www.pppl.gov 